\title{
Assessment of the use of latissimus dorsi restoration during modified radical mastectomy in breast cancer patients
}

\author{
CHAO YANG, XINLE WANG, GENG ZHANG, LIU YANG, XUAN GUO, XUE WANG and ZHENCHUAN SONG
}

Breast Center, the Fourth Hospital of Hebei Medical University, Shijiazhuang, Hebei 050011, P.R. China

Received March 13, 2017; Accepted September 28, 2017

DOI: $10.3892 / \mathrm{mco} .2017 .1435$

\begin{abstract}
The incision area of modified radical mastectomy in breast cancer patients is associated with subcutaneous effusion and skin flap necrosis as the most common complications. The aim of the present study was to assess the effect of latissimus dorsi restoration during modified radical mastectomy in breast cancer patients by evaluating 365 cases. Among these cases, 185 received modified radical mastectomy combined with intraoperative latissimus dorsi restoration, while 180 received modified radical mastectomy alone. The flap tension, drainage fluid amount and extubation time were compared between the two methods. The flap tension in patients who received modified radical mastectomy combined with intraoperative latissimus dorsi restoration was significantly lower and the blood supply was better. After surgery, the drainage fluid amount was also significantly lower in these patients compared with the control group. Furthermore, the mean time to drainage tube removal was significantly shorter in these patients and the postoperative cosmetic outcome was superior. Thus, it was concluded that modified radical mastectomy combined with intraoperative latissimus dorsi restoration in breast cancer patients was associated with reduced drainage fluid effusion, shorter hospitalization time and decreased flap tension. Overall, this method appears to be potentially suitable for extensive application in the clinical setting.
\end{abstract}

\section{Introduction}

Subcutaneous effusion and skin flap necrosis have been the most commonly reported complications at the incision area of modified radical mastectomy for breast cancer. Furthermore, the dissection of a partial latissimus dorsi muscle flap to clear the axillary lymph nodes may be associated with unsatisfactory aesthetic result and shape due to postoperative atrophy of

Correspondence to: Dr Zhengchuan Song, Breast Center, the Fourth Hospital of Hebei Medical University, 169 Tianshan Street, Shijiazhuang, Hebei 050011, P.R. China

E-mail: Songzhch@hotmail.com

Key words: breast cancer, modified radical mastectomy, latissimus dorsi restoration the latissimus dorsi muscle $(1,2)$. The aim of the present study was to evaluate the flap recovery, postoperative drainage fluid volume and time to drainage tube removal following latissimus dorsi restoration during modified radical mastectomy for breast cancer. The usefulness of this surgical method was investigated with respect to preoperative and postoperative complications, and the results are reported below.

\section{Patients and methods}

General characteristics. The patients who were included in our study were selected based on the following criteria: i) All the breast cancer patients had a single lesion; ii) the patients had no disorders of vital organs, such as the heart, brain, liver or kidney; and iii) the patients did not suffer from any additional conditions, such as diabetes, blood coagulation disorders or psychological problems.

Based on the abovementioned criteria, a total of 365 primary breast cancer patients who were treated at the Fourth Hospital of Hebei Medical University (Shijiazhuang, China) between April 2014 and April 2015, were enrolled in the present study and were randomly divided into two groups. The 185 patients in the experimental group received modified radical mastectomy combined with intraoperative latissimus dorsi restoration. The age of these patients ranged between 25 and 76 years (mean \pm standard deviation, $53.34 \pm 4.39$ years). In terms of TNM staging classification, 37 cases had stage I, 113 had stage II and 35 cases had stage IIIA disease. The control group included 180 patients who received modified radical mastectomy alone. These patients were aged 26-77 years $(54.71 \pm 5.26$ years). As regards TNM stage, 38 control patients had stage I, 112 had stage II and 30 had stage IIIA disease. There were no significant differences between the two groups regarding age or TNM stage $(\mathrm{P}>0.05)$. The clinical data are summarized in Table I. Following admission, all the patients or their family members signed an informed consent and operation agreement. The study protocol was sanctioned and supervised by the Medical Ethics Committee of the Fourth Hospital of Hebei Medical University.

All the patients underwent a modified radical mastectomy, and the procedures were performed by an experienced surgical team. The incision was performed layer by layer and the breast flap was dissected. The tissue $0.4 \mathrm{~cm}$ below the subcutaneous adipose layer was preserved and the blood supply by capillary networks was protected. During surgery, the epidermis 
overlying the tumor, breast tissue and pectoralis major fascia were resected. An incision was made in the armpit parallel to the anterior axillary fold, and the axillary lymph nodes were dissected. The thoracodorsal vascular pedicle of the latissimus dorsi was protected (3), as shown in Fig. 1. Following modified radical mastectomy, the patients in the experimental group received latissimus dorsi restoration. The leading edge of the latissimus dorsi was sutured with 3-5 stitches along the original attachment to be restored and fixed on the serratus anterior muscle; the original point of attachment of the latissimus dorsi to the serratus anterior is shown in Fig. 2.

Observational index. The surgical incisions were inspected daily after surgery. During these inspections, the patients were examined for presence of flap necrosis or subcutaneous effusion, along with the measurement of volume of drainage fluid, every day postoperatively. If there was no subcutaneous effusion, the drainage tube contained clear plasma-like liquid, and the volume of drainage fluid was $<15 \mathrm{ml}$, a decision was made to remove the drainage tubes. The changes in the shape of the latissimus dorsi were next compared and the clinical effect and breast appearance were assessed at 1 week, 1 month, 3 months and 6 months postoperatively.

Statistical methods. All the data were analyzed using statistical analysis software, SPSS 19.0 (SPSS Inc., Chicago, IL, USA). The quantitative data (mean \pm standard deviation) were analyzed using the t-test and the percentages were calculated using the $\chi^{2}$ test. P-values $<0.05$ were considered to indicate statistically significant differences.

\section{Results}

Comparison of drainage fluid volume. The comparison of the amount of drainage fluid between the two groups after surgery revealed that the experimental group patients exhibited significantly lower volumes of drainage fluid compared with the control group at 1,2, 3 and 4 days postoperatively $(\mathrm{P}<0.05$; Table I).

Comparison of time to drainage tube removal. The mean time to drainage tube removal in the experimental group was $4.61 \pm 1.78$ days, compared with $6.21 \pm 2.43$ days in the control group; the difference was statistically significant $(\mathrm{P}<0.05)$.

Cosmetic outcome. In addition, the patients who received intraoperative latissimus dorsi restoration exhibited a better shape compared with those who did not undergo restoration (Figs. 3 and 4).

Comparison of flap skin ischemia and necrosis. Flap skin ischemia and necrosis were observed in 5 cases $(5 / 185,2.70 \%)$ in the experimental group and 17 cases $(17 / 180,9.44 \%)$ in the control group $(\mathrm{P}<0.05)$. Furthermore, 3 cases $(3 / 112,2.68 \%)$ in the experimental group exhibited necrosis with a transverse incision, while 5 such cases $(5 / 111,4.5 \%)$ were observed in the control group $(\mathrm{P}=0.499)$. However, 2 cases $(2 / 73,2.74 \%)$ in the experimental group exhibited necrosis with a vertical incision, whereas 12 such cases $(12 / 69,17.39 \%)$ were observed in the control group $(\mathrm{P}<0.05)$.

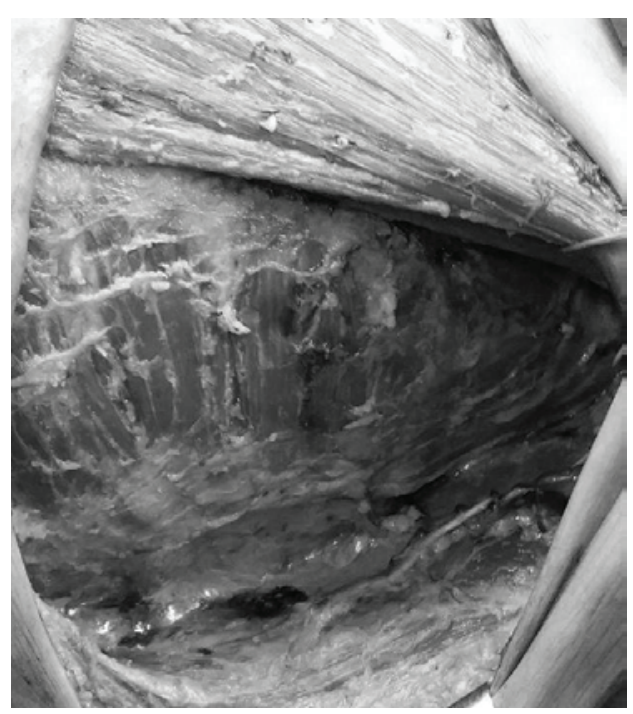

Figure 1. Latissimus dorsi dissection during modified radical mastectomy for breast cancer.

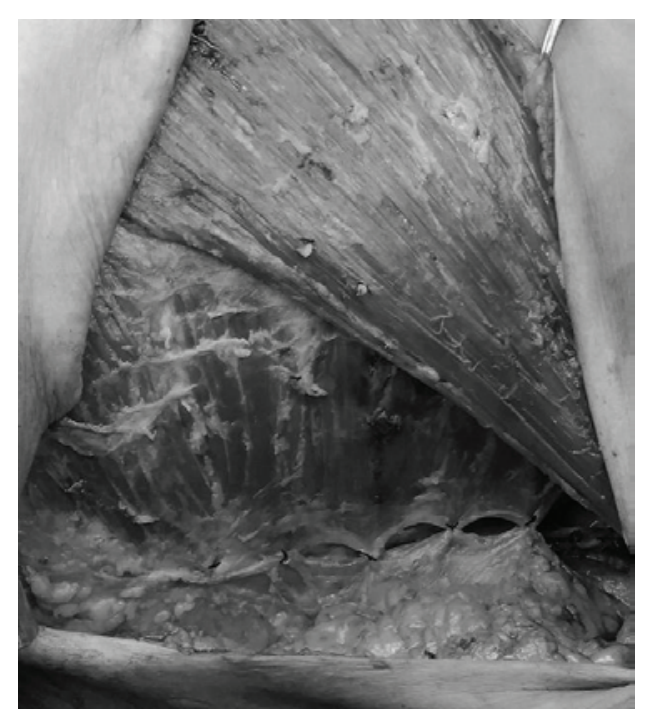

Figure 2. Latissimus dorsi restoration in modified radical mastectomy for breast cancer.

\section{Discussion}

Modified radical mastectomy is currently the preferred surgical method for the treatment of breast cancer (4). The resection of the mammary gland and axillary fat tissue usually results in the creation of a large space between the breast flap and the chest wall and axilla (5). The main causes of postoperative suboptimal healing are as follows: Inability to achieve complete intraoperative hemostasis, unligated macro-lymphatics, postoperative fat liquefaction, effusion underneath the flap and its insufficient drainage (6). Currently, the electrotome is commonly used to perform modified radical mastectomy for breast cancer, to isolate the flap and remove the axillary lymph nodes; however, it leads to bleeding and lymphatic fistula during and after surgery, and increases the drainage fluid volume, thus requiring a longer recovery time (12.5 53.6 days). Alternatively, the utilization of the ultrasound knife to remove the axillary lymph nodes may shorten the recovery time, 
Table I. Comparison of the drainage fluid amount (ml) between the two groups.

\begin{tabular}{lcccc}
\hline & \multicolumn{3}{c}{ Days } \\
\cline { 2 - 5 } Groups & First & Second & Third & Fourth \\
\hline Experimental & $135.97 \pm 17.37$ & $81.73 \pm 10.03$ & $52.22 \pm 14.25$ & $14.69 \pm 6.91$ \\
Control & $173.94 \pm 16.11$ & $98.33 \pm 11.36$ & $69.02 \pm 11.88$ & $30.91 \pm 8.12$ \\
$\mathrm{t}$ & 4.9385 & 2.6641 & 2.9784 & 3.8046 \\
P-value & $<0.05$ & $<0.05$ & $<0.05$ & $<0.05$ \\
\hline
\end{tabular}

Values are presented as mean \pm standard deviation.

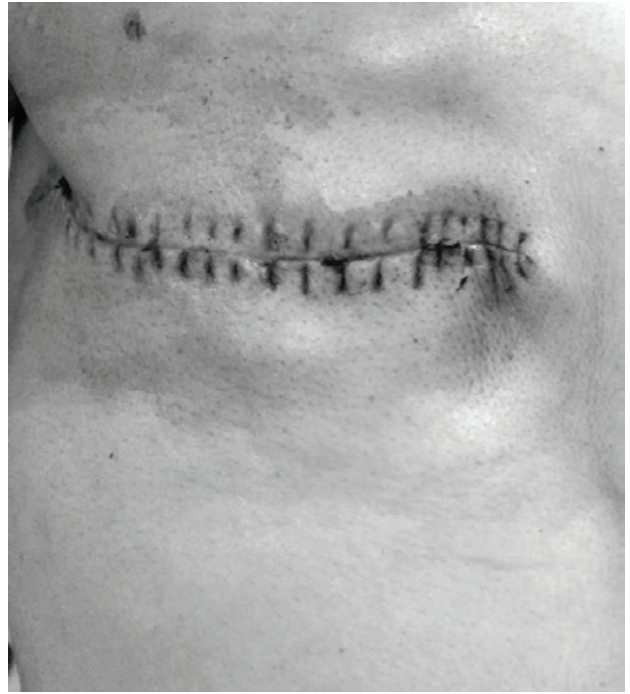

Figure 3. Appearance of the breast following intraoperative latissimus dorsi restoration during modified radical mastectomy.

although it remains relatively long (7.1 \pm 2.3 days) (7). During intraoperative clearing of the axillary lymph nodes, the anterior border of the latissimus dorsi must be resected and isolated along the serratus anterior muscle. If the restoration is not performed, the anterior border of the latissimus dorsi must be repositioned at $3-5 \mathrm{~cm}$ posteriorly, and the contracture in this site may lead to an unsatisfactory aesthetic result and appearance, increasing patient discomfort and adversely affecting the cosmetic outcome (8). Intraoperative latissimus dorsi restoration may recover the original anterior border of the latissimus dorsi, close the residual cavity in the axillary region, and accelerate the healing of the axillary lymphatics, reduce the amount of the effusion and shorten postoperative recovery time.

In addition, part of the skin overlying the tumors must also be resected in modified radical mastectomy for breast cancer. Generally, the transverse fusiform incision in the chest wall may mitigate the flap tension, which occurs due to longitudinal or other surgical incisions performed according to the locations of the breast lesion(s). The high flap tension may lead to the non-adhesion of the flap in the sagging area of the chest wall, particularly the axillary region, and finally induce skin flap edema and skin flap necrosis $(9,10)$. Several factors may lead to skin flap necrosis postoperatively, such as

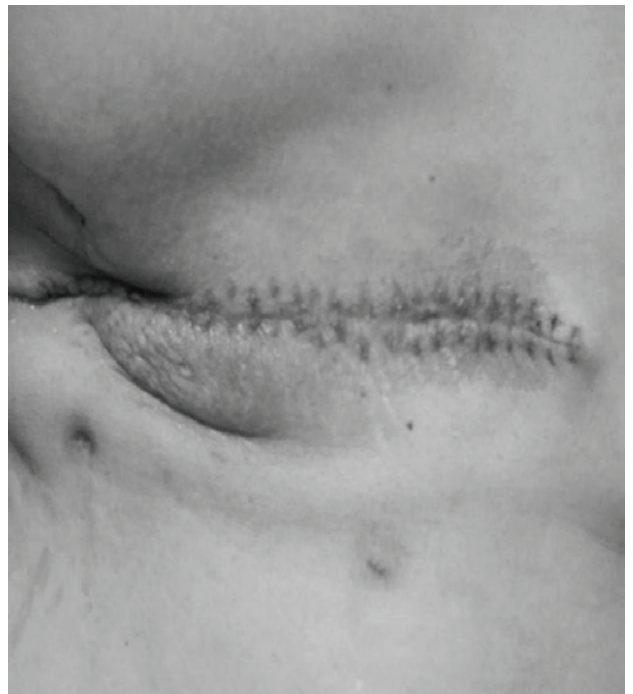

Figure 4. Appearance of the breast without intraoperative latissimus dorsi restoration during modified radical mastectomy.

the location of the incision, the thickness of the free flap, the flap tension, subcutaneous effusion, electric knife burns and infections (11-13). Ming et al (14) performed a comparison and observed that the skin flap necrosis rate due to transverse fusiform incision (13.5\%) postoperatively was significantly lower compared with that with the longitudinal incision method (28.9\%) in modified radical mastectomy for breast cancer. Therefore, it appears that by simply minimizing the skin flap tension, the patients recover faster. Thus, with intraoperative latissimus dorsi restoration, the high tension of the skin flap was reduced, subsequently resulting in relaxation of bilateral skin flap involution and lowering the incidence of skin flap necrosis caused by reduced blood supply. Therefore, latissimus dorsi recovery may reduce drainage fluid seepage and the risk of ischemia and necrosis of the skin flap, particularly in cases where the vertical incision is applied.

During latissimus dorsi resection for removing the axillary lymph nodes, patients usually feel uncomfortable when lying on the operated side postoperatively, due to the retraction of the anterior border of the latissimus dorsi, which appears to assume an uneven shape and is sensitive to touch. Moreover, the edges of the scar may be pulled apart during postoperative functional exercises, and the functional recovery of the affected limb is slow (15). In the experimental group, intraoperative 
latissimus dorsi restoration was performed following modified radical mastectomy for breast cancer. The flap tension in the experimental group patients was lower compared with that of patients in the control group. Consistent with this observation, high flap tension was also observed in patients who received a longitudinal incision, and this high tension may adversely affect the blood supply of the flap. Patients who received intraoperative latissimus dorsi restoration and removed the pressure dressing postoperatively, were more satisfied with the cosmetic outcome compared with those who did not receive this restoration. Furthermore, the breast appearance was assessed at 6 months postoperatively, and patients in the experimental group were satisfied with the cosmetic result. Moreover, there was no shrinkage of the latissimus dorsi or foreign body sensation upon touching. There was a definitive improvement in the patient's quality of life. The additional comparison of the clinical effect and breast appearance between the two groups at 6 months postoperatively led to the observation that the experimental group patients exhibited better clinical efficacy and superior cosmetic effect. However, latissimus dorsi restoration may cause adhesion between the latissimus dorsi and serratus anterior muscle and delay breast reconstruction.

In conclusion, our study demonstrated that modified radical mastectomy combined with intraoperative latissimus dorsi restoration method in breast cancer patients may significantly decrease drainage fluid effusion, shorten hospitalization time, reduce intraoperative flap tension, and decrease the risk of skin flap necrosis. The clinical effectiveness and patients' satisfaction with the cosmetic outcome were also improved. Thus, this method appears to be suitable for wider application in the clinical setting.

\section{References}

1. Han Z, Zhou Y and Han K: Breast cancer radical mastectomy preserving intercostobrachial nerve: Improve the quality of life for postoperative patients. Chin J Clin Anat 29: 591-593, 2011.
2. Wang Y, Chen J and Chen B: Application of extended latissimus dorsi flap in immediate breast reconstruction after modified radical mastectomy. Chin J Bases Clin Gen Surg 17: 735-738, 2010.

3. Mo J and Tan S: Immediate breast reconstruction with the latissimus dorsimuscle flap after modified radical mastectomy for breast cancer. Pract J Cancer 28: 357-359, 2013.

4. Staradub VL and Morrow M: Modified radical mastectomy with knife technique. Arch Surg 137: 105-110, 2002. (J).

5. Zhang H, Wang Y, Peng D, et al: Nipple-areola complex sparing modified radical mastectomy for breast cancer: Report of 159 cases. Chin J Gen Surg 29: 751-754, 2011.

6. Zhong T, Hofer SO, McCready DR, Jacks LM, Cook FE and Baxter N: A comparison of surgical complications between immediate breast reconstruction and mastectomy: The impact on delivery of chemotherapy--an analysis of 391 procedures. Ann Surg Oncol 19: 560-566, 2012

7. Wang D, Tang J, Qin J, et al: Application and evaluation of harmonic scalpel in breast cancer surgery axillary lymph node dissection. Chin J Surg Oncol 7: 30-32, 2015.

8. Cao Z, Liu H and Chen J: Immediate breast reconstruction in 24 patients after Nipple-sparing modified radical mastectomy of breast cancer. Chin J Clin Oncol 37: 104, 2010.

9. Yan X, Hu Y and Cui R: Clinical analysis of preserving intercostobrachial nerves in modified radical mastectomy for breast cancer. Chin Arch Gen Surg 9: 37-39, 2015. (Electronic Edition).

10. Phillips BT, Lanier ST, Conkling N, Wang ED, Dagum AB, Ganz JC, Khan SU and Bui DT: Intraoperative perfusion techniques can accurately predict mastectomy skin flap necrosis in breast reconstruction: Results of a prospective trial. Plast Reconstr Surg 129: 778e-788e, 2012. (J).

11. Bayram Y, Kulahci Y, Irgil C, Calikapan M and Noyan N: Skinreducing subcutaneous mastectomy using a dermal barrier flap and immediate breast reconstruction with an implant: A new surgical design for reconstruction of early-stage breast cancer. Aesthetic Plast Surg 34: 71-77, 2010. (J).

12. Garvey EM, Gray RJ, Wasif N, et al: Neoadjuvant therapy and a closer look at postoperative complication. Am J Surg 206: 894-899, 2013. (J).

13. Ribeiro GHFP, Kerr LM, Haikel RL, et al: Modified radical mastectomy: Apilot clinical trail comparing the conventional. Int J SurgeryLond Engl 11: 496-500, 2013.

14. Ming L, Liu H and Li J: An analysis of transverse incisional and longitudinal incision in modified radical mastectomy of breast cancer. Mod Oncol 18: 1765-1767, 2010.

15. Tan Y, Zhou E and He D: The clinical significance of reserving intercostobrachial nerve in modified radical mastectomy for breast cancer. Chin J Mod Operative Surg 14: 33-35, 2010. 